\title{
ESTUDIO SOBRE LA EFICACIA DEL RÉGIMEN FISCAL COOPERATIVO PORTUGUÉS
}

\author{
POR \\ Nina AGUIAR ${ }^{1}$ \\ Deolinda MEIRA ${ }^{2}$ y \\ Sandra RAQUEL ${ }^{3}$
}

\section{RESUMEN}

El ordenamiento jurídico portugués consagra un régimen fiscal especial para el sector cooperativo, basado, al igual que otros ordenamientos como el español o el italiano, en la protección de la mutualidad como forma de organización empresarial especialmente benéfica en el plano social. Para alcanzar ese objetivo, el régimen fiscal cooperativo debe ser selectivo, lo que significa que al legislador se le plantea el reto de establecer criterios para separar, dentro del marco cooperativo, lo que debe ser protegido de lo que no merece protección fiscal. El legislador portugués optó por un modelo basado en dos grupos de ramos cooperativos claramente diferenciados según los beneficios fiscales aplicables, ambos con amplias exenciones fiscales. El presente trabajo no se centra en el contenido de los beneficios aplicables sino en las condiciones que las cooperativas deben reunir para acogerse a esos regímenes fiscales favorables. Estos criterios son: i) una división entre operaciones con socios y operaciones con terceros; ii) una delimitación de las operaciones o actividades cooperativas según estén o no vinculadas con el "fin propio de la cooperativa"; y iii) una estructura prevalentemente mutualista del factor trabajo. Esta fórmula legal tiene su raíz en una legislación de 1929 y se ha mantenido hasta el día de hoy debido en parte a un fenómeno de inercia legislativa. El presente trabajo, basándose en la metodología de la sociología jurídica, asienta en una encuesta dirigida a 64 cooperativas, por la que se buscaba indagar hasta qué punto estos criterios (de acuerdo con los que se seleccionan las cooperativas que pueden acogerse a los regímenes fiscales favorables) cuadran con la realidad cooperativa actual.

\footnotetext{
${ }^{1}$ Investigadora del CECEJ (Centro de Estudos em Ciências Empresariais e Jurídicas); Profesora adjunta del Instituto Politécnico de Bragança. Dirección de correo electrónico: naguiar@ipb.pt.

${ }^{2}$ Investigadora del CECEJ (Centro de Estudos em Ciências Empresariais e Jurídicas); Profesora Adjunta del Instituto Politécnico do Porto. Dirección de correo electrónico: dl.meira@sapo.pt.

${ }^{3}$ Investigadora del Instituto Politécnico do Porto.

REVESCO Nº 121 - Segundo Cuatrimestre 2016 - ISSN: 1885-8031 - www.ucm.es/info/revesco

http://dx.doi.org/10.5209/rev_REVE.2016.v121.51306
}

Fecha de recepción: 31/08/2014

Fecha de aceptación: 08/09/2015 
Como era de esperar, la vetustez del régimen hizo que se encontraran desajustes muy significativos, que reclaman una reforma urgente.

Palabras clave: Cooperativas; mutualidad; exenciones fiscales; criterios de selectividad; adecuación.

Claves Econlit: H390, H220, H250.

\title{
STUDY ON THE EFFICACY OF THE PORTUGUESE COOPERATIVE TAXATION
}

\begin{abstract}
The Portuguese legal system encompasses a special tax regime for cooperative societies based, like many other legal systems like the Spanish or the Italian systems, on the protection of mutualism as a business organization form with particular social advantages. In order to achieve that goal the cooperative tax regime must be selective; in other words, the legislator must find objective criteria to distinguish cooperatives that deserve tax protection from those that do not. The Portuguese model is based on a main division of cooperatives into two groups according to their activity sector. Both the exemptions and the eligibility requisites are quite different for the two groups. This paper deals with the requirements to be met by cooperatives of both groups in order to be granted tax exemptions, analyzing their adequacy to present conditions. Eligibility criteria take into account: i) operations with third parties and with members; ii) income-generating operations related or unrelated to the specific activity of the cooperative; and iii) the existence of a mainly mutualistic structure of labor relations. This model stems from a 1929 law still in force because of legislative inertia. This paper is based on a survey carried out in 64 cooperatives, aimed at determining whether the described criteria (used for selecting those cooperatives that may benefit from tax exemption) are adequate to the present cooperative reality. As expected, significant shortcomings have been found due to the application of an outdated regime, calling for urgent reform.
\end{abstract}

Keywords: Cooperatives, mutualism, tax shelters, eligibility criteria; adequacy. 


\section{INTRODUCCIÓN}

\subsection{Objetivos y métodos}

El presente trabajo tiene los siguientes objetivos: Primero: responder a la pregunta sobre si el actual régimen fiscal cooperativo portugués logra realizar su finalidad principal, que consiste en proteger e incentivar el modelo de funcionamiento de las cooperativas basado en la mutualidad; Segundo: Caso se concluya que el régimen fiscal portugués actual no logra proteger e incentivar el modelo de funcionamiento de las cooperativas basado en la mutualidad, identificar si la raíz de esa ineficacia tiene un carácter conceptual, relacionado con la manera en que la normativa fiscal traza la divisoria entre el modelo mutualista y el modelo no mutualista; Tercero: Caso se concluya que la raíz de la hipotética ineficacia del régimen fiscal cooperativo portugués reside en la falla conceptual descrita, identificar posibles puntos que puedan ser mejorados; Cuarto: Caso se concluya que la raíz da la hipotética ineficacia del régimen fiscal cooperativo no reside, o no reside únicamente, en las mencionadas fallas conceptuales sino también en problemas de técnica fiscal, identificar estos problemas y formular propuestas para una solución a corto plazo.

El estudio se enmarca metodológicamente tanto en el campo del derecho (derecho tributario y derecho mercantil), como en el de la sociología del derecho, con una predominancia de esta última. La parte empírica del trabajo se basó en una encuesta llevada a cabo en 64 cooperativas de todos los ramos cooperativos previstos en derecho portugués ${ }^{4}$. La muestra seleccionada originalmente era de 818 cooperativas, repartidas por todo el territorio portugués y manteniendo la proporción de cada ramo cooperativo en la población total de cooperativas existentes en Portugal, que es de $2260^{5}$. Al final del trabajo de campo, realizado entre mayo y julio de 2014, se obtuvieron 64 cuestionarios completos y válidos.

\footnotetext{
${ }^{4}$ En Portugal existen doce ramos cooperativos, admitiéndose, expresamente, que una cooperativa pueda ejercer actividades propias de varios ramos (art. 4 del Código Cooperativo). Los distintos ramos cooperativos están regulados en legislación propia: Decreto-Ley 335/99, de 20 de agosto (cooperativas agrícolas); Decreto-Ley 523/99, de 10 de diciembre (cooperativas de comercialización); Decreto-Ley 522/99, de 10 de diciembre (cooperativas de consumo); Decreto-Ley 24/91, de 11 de enero, con las modificaciones del Decreto-Ley 230/95, de 12 de setiembre; Decreto-Ley 320/97, de 25 de noviembre; Decreto-Ley 102/99, de 31 de marzo; y Decreto-Ley 142/2009, de 16 de junio (cooperativas de crédito); Decreto-Ley 313/81, de 19 de noviembre (cooperativas culturales); Decreto-Ley 441-A/82, de 6 de noviembre (cooperativas de enseñanza); Decreto-Ley 502/99, de 19 de noviembre (cooperativas de vivienda y construcción); Decreto-Ley 312/81, de 18 de noviembre (cooperativas pesqueras); Decreto-Ley 309/81, de 16 de noviembre (cooperativas de producción operaria); Decreto-Ley 323/81, de 4 de diciembre (cooperativas de servicios); Decreto-Ley 7/98, de 15 de enero (cooperativas de solidaridad social).

${ }^{5}$ Se utilizó el directorio del Cases - Instituto Antonio Sérgio para a Economia Social. Los datos sobre el número total de cooperativas provienen del INE - Instituto Nacional de Estatística.
} 
Consideramos que la muestra obtenida $(2,8 \%)$ es representativa, ya que refleja la distribución de la población nacional tanto por ramos cooperativos como en el territorio nacional, con la salvedad de que los resultados obtenidos deben considerarse una primera aproximación cuantitativa a la realidad socio-jurídica que se pretende caracterizar.

Se buscó indagar sobre el cumplimiento, por parte de las cooperativas, de los criterios en los que asienta la concesión a estas entidades del régimen de beneficios fiscales que la normativa tributaria establece en su favor. Resulta importante resaltar que algunos de dichos criterios, que se describirán más adelante, son únicamente condiciones para la aplicación de los beneficios fiscales cooperativos, mientras otros resultan de obligado cumplimiento al amparo del derecho cooperativo sustantivo.

\section{LA PROBLEMÁTICA DE UN RÉGIMEN FISCAL COOPERATIVO DIFERENCIADO}

\subsection{Las cooperativas como organizaciones empresariales mutualistas}

De la definición de empresa como una organización de factores productivos capaz de producir bienes o servicios destinados a su oferta mediante remuneración, con vista a la obtención de un excedente del precio de venta sobre el coste (Abreu, 2012: 211), se desprende que las cooperativas son organizaciones empresariales (Meira, 2009: 43-50; Abreu, 2012: 281; Alguacil Marí, 2010: 31).

Ateniéndonos por otra parte al concepto jurídico de lucro, que consiste en un incremento del patrimonio (Abreu, 2012: 283), conseguido mediante una actividad económica, y asumiendo que el ánimo de lucro significa el propósito de realizar ese incremento patrimonial, puede por tanto afirmarse que las cooperativas no tienen dicho ánimo de lucro, sino principalmente un fin mutualista ${ }^{6}$, ya que su finalidad no consiste en el aumento de su patrimonio ni del patrimonio de sus socios (tras la distribución de un lucro en la forma de dividendo), sino en proporcionar a los socios una ventaja económica que se produce directamente en su patrimonio al realizarse la actividad cooperativa (Abreu, 2012: 281). Además, tal ventaja económica se obtiene a través del ejercicio de una actividad llevada a cabo por la cooperativa con los socios (Abreu, 2012: 283; Meira, 2012a: 358-360). Lo dicho

\footnotetext{
${ }^{6}$ La cooperativa es una empresa que, como cualquier otra, se dedica a la producción de bienes o a la prestación de servicios, y cuyo propósito consiste en maximizar ganancias y minimizar costes. La cooperativa no es, por tanto, una «obra de beneficencia» o una «institución de caridad» (Verrucoli, 1962: 550), sino una organización empresarial con una finalidad notoriamente económica (Boettcher, 1981).
} 
resulta aplicable a todo tipo de cooperativa, cualquiera sea su actividad, y es válido incluso para las cooperativas de solidaridad social que, en la acepción descrita, son también organizaciones empresariales (Meira, 2013b: 30; Abreu, 2012: 282).

Para resumir en pocas palabras la compleja esencia de las cooperativas, se trata de organizaciones empresariales que, a diferencia de las sociedades mercantiles: i) no tienen ánimo de lucro, sino que su objetivo es obtener ventajas económicas producidas directamente en el patrimonio de los socios; ii) dichas ventajas económicas producidas directamente en el patrimonio de los socios resultan del ejercicio de una actividad económica llevada a cabo por la cooperativa y en la que los socios deben participar (actividad cooperativizada) (Vargas Vasserot, 2006). Podría afirmarse entonces, como ya lo hacen algunas concepciones, que son los socios quienes verdaderamente realizan la actividad cooperativa, utilizando la cooperativa como un mero instrumento jurídico para esa realización (Chambers, 1947: 2). Por esta razón, el socio que no ejerza la actividad cooperativa no obtiene, en principio, ninguna ventaja económica. La conjunción de estos dos factores - tener como finalidad obtener ventajas económicas producidas directamente en el patrimonio de los socios, a través de un modo particular de ejercicio de una actividad que asienta en la participación activa de los socios en la actividad económica (como adquiriente de bienes o servicios a la cooperativa, como suministrador de bienes o servicios a la cooperativa, o bien como prestador de trabajo en el ámbito de la actividad cooperativa) - es lo que caracteriza el modelo mutualista de funcionamiento de las cooperativas (Vargas Vasserot, 2006). Y en correspondencia con el modelo de funcionamiento descrito, también el ánimo o finalidad de las cooperativas debe ser entendido como un ánimo mutualista (Meira, 2012a).

\subsection{La tributación de las cooperativas - perspectiva general}

Con respecto a la tributación de las cooperativas, se pueden identificar dos tendencias principales al comparar los sistemas jurídicos europeos continentales con los anglosajones. En los primeros, la concepción predominante es que, al contrario de las sociedades mercantiles, las cooperativas cumplen una función social de gran relevancia y por esa razón deben gozar de una protección especial (Fajardo García, 2013; Hinojosa Torralvo, 2010), incluyendo una protección fiscal ${ }^{7}$. En los segundos, de los que son ejemplos los Estados Unidos de América,

\footnotetext{
${ }^{7}$ El principio de protección del sector cooperativo y social [pár. f) del art. 80 de la Constitución de la República Portuguesa] fundamenta bien las discriminaciones positivas que el legislador concede al sector, bien como la previsión de medidas materiales que permitan su desarrollo (Meira, 2013b: 42-45). En consonancia con este principio, el art. 85 de la CRP consagra, en su núm. 1, el estímulo y el apoyo a la creación y a la actividad de la
} 
Reino Unido ${ }^{8}$ y Canadá, ${ }^{9}$ la concepción general es que las cooperativas requieren un trato fiscal especial porque los ingresos o ganancias de las cooperativas no forman verdaderamente una renta de las cooperativas sino de sus socios (Petrou, 2013: 311; Chambers, 1947: 99).

Como se deduce de inmediato, los problemas conceptuales que plantean las dos concepciones son distintos, aunque terminen solapándose con frecuencia. Este artículo se ocupa, naturalmente, de la concepción que afecta sobre todo a los países de Europa continental. Y dentro de ésta, se toma como referencia el régimen fiscal cooperativo portugués que, a su vez, presenta bastantes especialidades cuando se lo compara con otros sistemas próximos, como el español o el italiano (Aguiar, 2014). Sin embargo, primeramente se exponen en breves líneas los marcos conceptuales de la protección fiscal de las cooperativas.

\subsection{Marcos conceptuales de la protección fiscal de las cooperativas}

Se ha mencionado la existencia de dos concepciones principales en lo concerniente a la tributación de las cooperativas, una de las cuales adoptada en los sistemas anglosajones, la otra en los sistemas europeos continentales. Para la primera, el régimen fiscal especial de las cooperativas estaría justificado por el hecho de que el resultado contable de las cooperativas no es una renta de la cooperativa (Ish, 1975; Pigou, 1920: 161), sino de los socios. Para la segunda, el régimen fiscal especial de las cooperativas estaría justificado por una necesidad de protección de las cooperativas, teniendo en cuenta su función social. En términos teóricos, la consecuencia inmediata de la puesta en práctica de cada una de estas concepciones resulta evidente: para la segunda concepción, el régimen fiscal especial de las cooperativas asienta en la dogmática de los beneficios fiscales. Para cada aspecto del régimen fiscal de las cooperativas hay que averiguar, en primer lugar, en qué medida se trata de un beneficio fiscal, y en segundo, si tal beneficio fiscal se justifica y es el adecuado (Tipke \& Lang, 2005: 808). Al contrario, la segunda concepción se sustrae al campo de los beneficios fiscales y debe analizarse de acuerdo con el principio de capacidad contributiva. Donde no existe capacidad contributiva no deberá haber lugar a tributación (Ish, 1975: 45), con lo que, si las cooperativas no tienen lucros, no puede aplicarse imposición alguna sobre la renta.

cooperativa; y en su núm. 2, garantiza que «la ley definirá los beneficios fiscales y financieros de las cooperativas, y bien así las condiciones más favorables para la obtención de crédito y apoyo técnico».

${ }^{8}$ Sobre el régimen fiscal cooperativo australiano, Snaith, 2013, 735-737.

${ }^{9}$ Sobre el régimen fiscal cooperativo canadiense, Petrou, 2013, 311-313. 
En lo que respecta a la concepción vigente en Portugal y en la mayoría de los países europeos continentales, donde el régimen fiscal de las cooperativas constituye un instrumento de protección del sector cooperativo, la teoría sobre las bases conceptuales de la protección fiscal de las cooperativas requiere aún una elaboración exhaustiva ${ }^{10}$. Pero si nos atenemos a la idea anteriormente expuesta, de que cualquier régimen fiscal de las cooperativas dentro de las concepciones "proteccionistas" debe ajustarse a la dogmática de los beneficios fiscales, se nos ofrece un conjunto de elementos jurídicos que deben tomarse como soporte de esa concepción, dado que ya han sido ampliamente validados por la doctrina justributarista.

El primer marco jurídico que hay que tener en cuenta es el principio constitucional de igualdad tributaria, que se opone a cualquier discriminación negativa o positiva en materia tributaria (Rodrigo Ruiz, 2003: 202; Nabais, 1998: 638). Sin embargo, el principio de igualdad tributaria no resulta incompatible con la existencia de beneficios fiscales (Tipke\&Lang, 2005: 807), si bien plantea exigencias estrictas para la creación de beneficios fiscales. Según el principio de igualdad tributaria, sólo puede aprobarse un beneficio fiscal si este tiene por finalidad proteger un bien social (Tipke \& Lang, 2005: 807) con un valor jurídico equivalente al principio de igualdad tributaria, lo que implica que deba tratarse de un valor constitucionalmente reconocido (Rodrigo Ruiz, 2003: 204) o con fundamento constitucional; en segundo lugar, el beneficio fiscal tiene que ser necesario y adecuado (Tipke \& Lang, 2005: 808) para alcanzar el objetivo deseado, que consiste en la protección del bien social elegido (Tipke \& Lang, 2005: 808), y tiene que ser proporcional, o sea, no sobrepasar la medida necesaria para alcanzar el objetivo deseado (Pereira et al. 1998: 28). Las exigencias descritas podrían formar en su conjunto el segundo marco jurídico que hay que tener en cuenta a la hora de establecer un beneficio fiscal.

El tercer marco jurídico que habría que considerar al analizar un régimen fiscal cooperativo favorable es un aspecto bastante debatido en fechas recientes y sobre el que existe un consenso mucho menor. Nos referimos al problema de la interacción entre los regímenes fiscales cooperativos favorables y las reglas europeas sobre ayudas de estado. ${ }^{11}$ En el caso portugués, el problema ha sido tratado en el plano legislativo al amparo del principio de neutralidad de los beneficios fiscales (Pereira et al.1998: 28).

\footnotetext{
${ }^{10}$ Se llama la atención, sin embargo, sobre la existencia de varios estudios que tratan aspectos conceptuales del régimen fiscal de las cooperativas, como, por ejemplo Calvo Ortega, 2005; Rodrigo Ruiz, 2003; o Elena Días, 1990, entre muchos otros.

${ }^{11}$ La bibliografía sobre el tema es extensa: Fajardo García, 2013; Cusa, 2013: 51-64; Rodrigo Ruíz, 2010; Alguacil Marí, 2010; Tejerizo López, 2010; Hinojosa Torralvo, 2010; Romero Civera, 2010.
} 


\subsection{El modelo de funcionamiento mutualista ("cooperativo") como objeto de protección fiscal}

Los regímenes fiscales cooperativos "proteccionistas" están justificados por la función social especialmente importante que realizan las cooperativas (Fajardo García; Stracke, 1997: 39; Hinojosa Torralvo, 2010: 77). Ahora bien, esta función social depende de que la cooperativa funcione en sintonía con el modelo mutualista. De cara a la emergencia de formas híbridas de cooperativas, que no se dirigen exclusivamente a la realización del fin mutualista sino al incremento de sus resultados financieros (Sarina, 2013: 226), el legislador fiscal tiene que establecer criterios cuya aplicación garantice que el régimen físcal cooperativo sólo beneficia, dentro de la actividad cooperativa, la zona de la mutualidad, excluyendo todo lo que, aún bajo la forma legal cooperativa, no se concilia con el fin mutualista.

Conviene por consiguiente recordar cuál es el núcleo del modelo de funcionamiento mutualista. Una primera característica consiste en que los socios de la cooperativa son los destinatarios principales de la actividad cooperativa (Namorado, 2000, 181-186). Aunque con personalidad jurídica propia $^{12}$, la cooperativa no tiene un fin autónomo con respecto a sus miembros (Duarte, 2012: 483-487), sino que consiste en un instrumento a través del cual los socios se entreayudan (cooperan) para la realización de sus propios intereses económicos. Una segunda característica reside en la obligación jurídica de los socios de participar en la actividad de la cooperativa (Meira, 2009: 212-220). Esta obligación se materializa de distintas maneras, dependiendo del objeto específico de la cooperativa y del sector de actividad (Vargas Vasserot, 2006: 105-198). Pero sea cual fuere ese modelo concreto, la participación del socio en la actividad cooperativa constituye siempre el mecanismo básico para el desarrollo del objeto social de la cooperativa y la obtención de la ventaja mutualista (Meira, 2012a: 369-273; Llobregat Hurtado, 1991: 20-46).

El modelo de funcionamiento mutualista tal como se acaba de describir tiene importantes consecuencias. La primera es que, si bien las operaciones económicas entre la cooperativa y los miembros por medio de las que se realiza el objeto cooperativo son esenciales a la mutualidad, ya las operaciones con terceros no lo son, aunque puedan resultar necesarias para garantizar la sostenibilidad empresarial de la cooperativa (Meira, 2012b). Otra consecuencia es que, asentando la mutualidad en la realización del objeto cooperativo mediante las relaciones de la cooperativa con los socios, si la cooperativa practica operaciones

${ }^{12}$ Arts. 2.1 y 16 del Código Cooperativo.

REVESCO No 121 - Segundo Cuatrimestre 2016 - ISSN: 1885-8031 - www.ucm.es/info/revesco 
económicas no directamente relacionadas con su objeto, esas operaciones serán necesariamente ajenas al ámbito de las relaciones mutualistas (Vargas Vasserot, 2006: 199227).

\section{EL RÉGIMEN FISCAL COOPERATIVO PORTUGUÉS}

\subsection{El estatuto fiscal general de las cooperativas}

En el derecho portugués las cooperativas son sujetos pasivos del impuesto sobre la renta de las personas jurídicas ${ }^{13}$, sin que se les reconozca allí ninguna especialidad. Además, la ley que regula dicho impuesto estipula que los "excedentes cooperativos" formen parte del resultado contable de la cooperativa, a efectos de calcular su beneficio imponible ${ }^{14}$.

Las cooperativas son igualmente sujetos pasivos de todos los demás impuestos que forman parte del sistema fiscal y en particular del Impuesto sobre el Valor Añadido (IVA), del Impuesto Municipal sobre Inmuebles (IMI), del Impuesto Municipal sobre las Transmisiones Inmuebles (IMT) y del Impuesto de Timbre.

\subsection{Los regímenes de exención - requisitos para su aplicación}

El régimen fiscal cooperativo portugués cuenta con un sistema de exenciones establecido al margen de los impuestos descritos, que abarca el impuesto sobre la renta, los impuestos sobre el patrimonio inmobiliario y sobre las transmisiones inmuebles y el impuesto de timbre ${ }^{15}$. Con el fin de garantizar que el régimen fiscal cooperativo actúa de una forma selectiva para proteger únicamente la zona de la mutualidad, la normativa portuguesa sigue un modelo distinto tanto del sistema español ${ }^{16}$ como del sistema italiano ${ }^{17}$. La normativa empieza por separar dos grandes grupos de ramos cooperativos (Aguiar, 2014). Un primer grupo, que en adelante se designará "Grupo A", incluye las cooperativas agrícolas, culturales, de consumo, de vivienda y construcción y de solidaridad social. El segundo grupo, al que en adelante llamaremos "Grupo B", incluye las cooperativas de comercialización, de crédito, de producción operaria, de artesanía, de pesca, de enseñanza y de servicios.

\footnotetext{
${ }^{13}$ Art. 2.1 del Código del Impuesto sobre las Personas Jurídicas.

${ }^{14}$ Artículo 17.2 del Código del Impuesto sobre las Personas Jurídicas.

${ }^{15}$ Art. 66-A del Estatuto de Beneficios Fiscales (EBF).

${ }^{16}$ Para una descripción del sistema fiscal cooperativo español, vd. Arana Landín, 2012.

${ }^{17}$ Sobre el sistema fiscal cooperativo italiano, $v d$. Dili, 2007.
} 
Los regímenes de exención para estos dos grupos no sólo dependen de la observancia de distintos requisitos, sino producen efectos muy diferentes. Conviene por ello entender la razón de esta división. La división de las cooperativas en estos dos grupos tiene su génesis en una legislación de $1929^{18}$ y se ha conservado, en nuestra opinión, por un fenómeno de inercia legislativa (Aguiar, 2014). Supuestamente, en el "Grupo A" se encontrarían los ramos cooperativos en los que las operaciones generadoras de ingresos se realizan normalmente con los socios, lo que significa, dicho de otro modo, que la mutualidad se lleva a cabo mediante unas relaciones en las que son los propios socios quienes adquieren los servicios o los bienes ofrecidos por las cooperativas. Por ejemplo, en una cooperativa de vivienda y construcción los ingresos de la cooperativa resultan, normalmente, de servicios prestados a los socios por la cooperativa. Los socios pagarán a la cooperativa un precio normalmente por debajo del precio de mercado, residiendo en esa diferencia (entre el precio de mercado y el precio pagado por el socio) la ventaja mutualista. En el "Grupo B" estarían los ramos cooperativos en los que las operaciones generadoras de ingresos se realizan normalmente entre la cooperativa y entidades que no son miembros (Aguiar, 2014), centrándose la mutualidad (la cooperación) en la prestación de trabajo por parte de los socios a las cooperativas En otras palabras, la mutualidad reside en la organización del trabajo de los socios y la ventaja mutualista del socio consiste en la remuneración, por encima de la remuneración vigente en el mercado, así como en su derecho de participar en la organización y en la oferta de su trabajo en el mercado.

Para el "Grupo A", el legislador traza una frontera entre mutualidad y "no mutualidad" similar a la que se establece en el régimen fiscal cooperativo español entre "resultados cooperativos" y "resultados no cooperativos"19 (Vargas Vasserot y Aguilar Rubio, 2004: 131). Esta línea divisoria queda definida gracias al concepto de "operaciones con terceros", que, según la doctrina cooperativista, son las operaciones realizadas entre la cooperativa y entidades no miembros dentro del objeto social de la cooperativa (Vargas Vasserot, 2006: 199-211; Meira, 2012b). Para el legislador fiscal, las “operaciones con terceros", en este grupo de cooperativas, no pertenecerían a la zona de la mutualidad y por ese motivo no beneficiarían de la exención fiscal. También en este primer grupo de ramos cooperativos, el

\footnotetext{
${ }^{18}$ Decreto 16731 , de 13 de abril de 1929.

${ }^{19}$ En las cooperativas pueden identificarse tres tipos principales de resultados: los resultados cooperativos, llamados excedentes, correspondientes a los resultados provenientes de la actividad económica ejercida entre la cooperativa y sus miembros (actividad cooperativizada); los resultados extracooperativos correspondientes a los resultados provenientes de las operaciones con terceros; y los resultados extraordinarios, provenientes de actividades ajenas al fin social de la cooperativa. [Esta distinción está expresamente prevista en la legislación cooperativa española (v.g. art. 57 de la Ley Estatal de Cooperativas - Ley 27/1999, de 16 de julio). Sobre esta distinción, vd.Gemma Fajardo, 1997: 120 ss; y Paniagua Zurera, 2005: 659-686.
} 
legislador delinea una segunda divisoria entre mutualidad y "no mutualidad" a través del concepto de "actividades ajenas a los fines propios de las cooperativas" 20 . Sin detenernos en el problema de la interpretación jurídica de esta norma (Aguiar, 2014), diremos únicamente que con esta segunda divisoria el legislador pretende excluir del ámbito de la protección fiscal las operaciones que no formen parte del núcleo del objeto social cooperativo, incluyendo las actividades previstas en los estatutos a título complementario (Aguiar, 2014). Por lo tanto, todas las actividades previstas en los estatutos, aunque no formen parte del objeto principal de la cooperativa, quedan dentro del perímetro de la exención, mientras las actividades no previstas en los estatutos quedarían fuera del ámbito de la mutualidad y, por consiguiente, del perímetro de la exención fiscal. De lo expuesto resulta que en este primer régimen de exención no se distingue entre cooperativas exentas y no exentas, sino entre operaciones exentas y no exentas.

Con respecto al régimen fiscal aplicable al Grupo B, hay que tomar en consideración que este régimen adopta como patrón las cooperativas de trabajo (producción operaria), en que los ingresos cooperativos son generados por medio de operaciones con entidades no miembros (clientes). Para este grupo de cooperativas, la frontera entre las zonas de mutualidad y de "no mutualidad" ya no radica en las características de las operaciones efectuadas por la cooperativa, sino en determinadas características de la propia cooperativa que pudieran funcionar como indicadores de su pureza mutualista (Cusa, 2013: 46-50). Así, para acogerse al régimen de exención, las cooperativas de este segundo grupo (Grupo B) tendrán que cumplir dos condiciones simultáneas: un $75 \%$ de los trabajadores de la cooperativa deberán ser socios y el $75 \%$ de los socios deberán ser trabajadores de la cooperativa. A fin de simplificar la terminología que se utiliza en el presente estudio, pasaremos a referirnos a este criterio como "estructura prevalentemente mutualista del factor trabajo". A diferencia del grupo anterior, en este segundo régimen (Grupo B), existen cooperativas exentas y cooperativas no exentas.

Además de los requisitos específicos establecidos para los dos grupos de ramos cooperativos mencionados, la normativa establece como condición general aplicable a toda cooperativa para que pueda acogerse a cualquiera de los regímenes de exención, que la cooperativa se encuentre "constituida, registrada y funcionando al abrigo del Código

\footnotetext{
${ }^{20}$ La legislación española habla en "fines específicos de la cooperativa” (art. 21.3 de la Ley 20/1990, de 19 de diciembre.
}

REVESCO No 121 - Segundo Cuatrimestre 2016 - ISSN: 1885-8031 - www.ucm.es/info/revesco 
Cooperativo" ${ }^{21}$. Del art. 2.1del Código Cooperativo resulta que el funcionamiento de las cooperativas asienta en la obediencia a los principios cooperativos enunciados en el art. 3 del mismo código, los cuales coinciden con los principios cooperativos proclamados por la Alianza Cooperativa Internacional, y que en el orden jurídico portugués tienen carácter vinculante por imposición constitucional ${ }^{22}$ (Meira, 2011). Del contexto descrito resulta que, en el ordenamiento jurídico portugués, el funcionamiento de las cooperativas se caracteriza por los principios de: la participación de los socios en la realización del objeto cooperativo; la gestión democrática de la cooperativa por parte de sus miembros; la orientación de la gestión hacia los miembros; la autonomía e independencia ante las autoridades públicas y otras entidades externas; y la transparencia, que se concretiza en el derecho de los socios a la información y en el poder de control y de fiscalización que ejercen la junta general y el consejo fiscal sobre el órgano de administración de la cooperativa (Meira, 2013a). Estos requisitos de funcionamiento de acuerdo con los principios cooperativos son igualmente importantes a la hora de garantizar que el régimen fiscal cooperativo proteja únicamente las entidades que funcionan según el modelo mutualista.

\section{ANÁLISIS EMPÍRICO}

\subsection{Estructura de la encuesta}

La encuesta llevada a cabo tuvo como primer propósito indagar hasta qué punto los requisitos descritos para que las cooperativas puedan beneficiar del régimen de exención fiscal se encuentran presentes en la realidad cooperativa portuguesa. En otras palabras, se trató de averiguar si las cooperativas existentes en la actualidad en Portugal funcionan predominantemente según un modelo mutualista, tal como la normativa fiscal lo entiende y como ya se ha puntualizado ${ }^{23}$. Obviamente, la concepción del modelo mutualista que plasma la normativa fiscal es, en algunos aspectos, un modelo mutualista simplificado, si tomamos como referencia toda la complejidad de la mutualidad cooperativa proyectada por el derecho sustantivo. Tal simplificación, sin embargo, es una característica necesaria y usual del derecho tributario, de cara a la complejidad de las realidades económicas, jurídicas y sociales que se abordan. Por ello, durante la lectura de este estudio debe tenerse siempre presente que no se busca contestar a la cuestión de si las cooperativas existentes funcionan según el modelo mutualista plasmado por el derecho cooperativo, sino únicamente averiguar si las

\footnotetext{
${ }^{21}$ Art. 66-A.13 del EBF - Estatuto de Beneficios Fiscales.

${ }^{22}$ Arts. 61. 2, y 82. 4, al. a), de la Constitución de la República Portuguesa (CRP).

${ }^{23}$ Ver el punto III. 2
} 
cooperativas en la actualidad tienden a adaptarse al modelo mutualista tal como este modelo fue traducido por la normativa fiscal.

Pueden proponerse tres situaciones hipotéticas preliminares. La primera, que las cooperativas tiendan a adaptarse al modelo mutualista según los criterios de la normativa fiscal, en cuyo caso puede considerarse que el régimen tributario, a pesar de su vetustez, conserva su eficacia como instrumento de protección de la mutualidad. La segunda, que las cooperativas tiendan a apartarse del modelo mutualista según los criterios de la normativa fiscal, en cuyo caso debería concluirse que el régimen fiscal ya no resulta eficaz en lo que concierne a la protección de la mutualidad cooperativa. La tercera, por último, que las cooperativas muestren una tendencia a atenerse formalmente al modelo mutualista según los criterios fiscales, pero que al mismo tiempo el fenómeno vaya acompañado de una “desmutualización” real. De confirmarse esta tercera hipótesis, habrá que concluir que el régimen fiscal no sólo no está adaptado para proteger la mutualidad cooperativa sino que, además, adolece de deficiencias técnicas que obstan a la eficacia del sistema, las cuales pueden también derivar de una obsolescencia del régimen fiscal frente a la realidad cooperativa actual.

A fin de verificar las hipótesis enumeradas, se plantearon las siguientes cuestiones:

A. ¿Las cooperativas definen su objeto y delimitan sus actividades de modo a que se pueda clasificar rigurosamente el sector de actividad en el que la cooperativa opera para efectos de aplicar la norma de exención fiscal?

B. ¿Qué importancia relativa tienen en los resultados de las cooperativas las operaciones ajenas a su objeto estatutario?

C. ¿Las cooperativas identifican separadamente en su contabilidad las operaciones que realizan con terceros?

D. ¿Qué parte de la actividad cooperativa representan las "operaciones con terceros"?

E. ¿Qué porcentaje de las cooperativas que forman el Grupo B cumplen el criterio de la "estructura prevalentemente mutualista del factor trabajo"? 


\subsection{Las actividades de las cooperativas y el objeto estatutario}

\subsubsection{La definición del objeto}

La definición del objeto de las cooperativas constituye uno de los aspectos clave para la aplicación del régimen de exenciones vigentes, por dos motivos principales, que se desprenden de lo dicho hasta aquí. Por una parte, el régimen de exenciones se basa en una división de las cooperativas según su ramo de actividad, lo que a su vez depende de cómo se define la actividad de cada cooperativa en los respectivos estatutos. Es decir que según cuál sea su objeto estatutario, la cooperativa quedará integrada en el "Grupo A" o en el "Grupo B". Y, por otra parte, el objeto de la cooperativa - la actividad empresarial que la cooperativa debe ejercer con los socios - constituye el punto de partida para definir las operaciones no conectadas con el "fin propio de las cooperativas" e incluso las "operaciones con terceros". Por consiguiente, para las cooperativas del "Grupo A", la definición del objeto estatutario constituye la base sobre la que se traza la frontera entre la mutualidad y la "no mutualidad", y entre las actividades exentas y no exentas.

Para analizar esta cuestión, dividimos las cooperativas según considerásemos que su objeto estaba definido de una forma abierta (cooperativas con un "objeto abierto") o que su objeto está definido de una forma cerrada (cooperativas con un "objeto cerrado"). Por "objeto abierto" entendemos una fórmula que deja totalmente abierto el abanico de actividades que la cooperativa puede realizar, de tal forma que ese objeto no queda delimitado por referencia a una actividad específica ni a un grupo de actividades específicas, ni siquiera a una complementariedad con el objeto principal. Se tuvo en consideración la necesidad de las cooperativas, como cualquier organización empresarial, de desarrollar actividades no estrictamente comprendidas en su objeto, pero que están conectadas con ese objeto, situaciones que no se incluyeron en lo que se ha definido como "objeto abierto". Como "objeto abierto" consideramos únicamente aquellas formulaciones constantes en los estatutos que no definen con precisión las actividades que la cooperativa puede desarrollar con carácter regular, dejando abierta la puerta para que la cooperativa pueda realizar cualquier actividad económica (sin entrar en el análisis de la licitud de las cláusulas de los estatutos de las 
cooperativas formuladas en los términos indicados, a luz del derecho cooperativo portugués $)^{24}$.

Las fórmulas utilizadas en la definición del objeto en los casos que consideramos "objeto abierto" pueden ser: "la cooperativa podrá subsidiariamente realizar actividades de otros sectores, necesarias para satisfacer las necesidades de sus miembros"; "promocionar o apoyar iniciativas de interés para los miembros"; "la creación y gestión de servicios de interés común"; "la cooperativa tiene como objeto la realización de acciones de apoyo al desarrollo integrado del territorio en el plano social, cultural y medioambiental, contribuyendo a la promoción de la región a través actividades de apoyo al turismo, artesanía y cultura, valorización de los recursos endógenos del territorio, estudios socioeconómicos y prestación de servicios de formación profesional y educación"; "la cooperativa tiene como objeto la prestación de servicios a sus miembros en el ámbito de la administración y la gestión de condominios y espacios comunes, de la vida privada y cotidiana de los miembros y de sus necesidades culturales, recreativas y deportivas"; "la satisfacción de las necesidades económicas, sociales o culturales de sus miembros"; "promocionar el fortalecimiento del sector de la economía social, profundizando la cooperación entre el Estado y las organizaciones que lo integran, con la finalidad de estimular su potencial al servicio del desarrollo socioeconómico del País"; "otras actividades propias y relativas a otros ramos del sector cooperativo"; "otras actividades complementarias o accesorias por deliberación de la Asamblea General".

La relevancia de la cuestión para el tema que nos ocupa resulta evidente. Las cooperativas con un "objeto abierto" pueden, en cualquier momento y con independencia del ramo cooperativo en que se encuadren, ejercer cualquier actividad generadora de rentas. Una vez que su objeto está definido de un manera abierta, a la administración físcal no le es posible definir el ramo o ramos cooperativos reales en que operan esas cooperativas, ni, a la vista de las actividades ejercidas durante un determinado año fiscal, determinar si dichas rentas generadas mediante tales actividades están conectadas con "el fin proprio de la cooperativa". Asimismo, la administración fiscal no tendría forma de delimitar con precisión

\footnotetext{
${ }^{24}$ Según al art. 11.2 del Código de las Sociedades Mercantiles, que se aplica subsidiariamente a las cooperativas por fuerza del art. 9 del Código Cooperativo, las sociedades deben definir de manera precisa la actividad o las actividades en que consiste el objeto societario, eliminado por esta vía la posibilidad de una indicación vaga o imprecisa de las actividades sociales.
} 
las "operaciones con terceros", dado que éstas son únicamente operaciones realizadas dentro del objeto de la cooperativa (Meira, 2012b).

En la muestra considerada de 64 cooperativas, 19 tienen un "objeto abierto", lo que significa un porcentaje superior al $31 \%$. Hay que concluir por tanto que el peso relativo de cooperativas con un "objeto abierto" (que pueden, según sus estatutos, desarrollar cualquier actividad económica) es muy relevante. Para las cooperativas del "Grupo A", la elevada expresión del fenómeno constituye un obstáculo significativo a la aplicación correcta del régimen fiscal cooperativo. Según sugieren estos datos, para el $31 \%$ de las cooperativas, ninguna operación puede considerarse ajena a sus "fines propios". Por ejemplo, si una cooperativa agrícola, "con un objeto abierto" ejerce la actividad de venta de materiales de construcción, esta actividad no podría considerarse, a efectos de la normativa fiscal, una actividad ajena al "fin propio" de la cooperativa, lo cual conllevaría la exclusión de las respectivas rentas del perímetro de la exención.

Figura 1. Distribución del "objeto abierto" y del "objeto cerrado" por ramos cooperativos

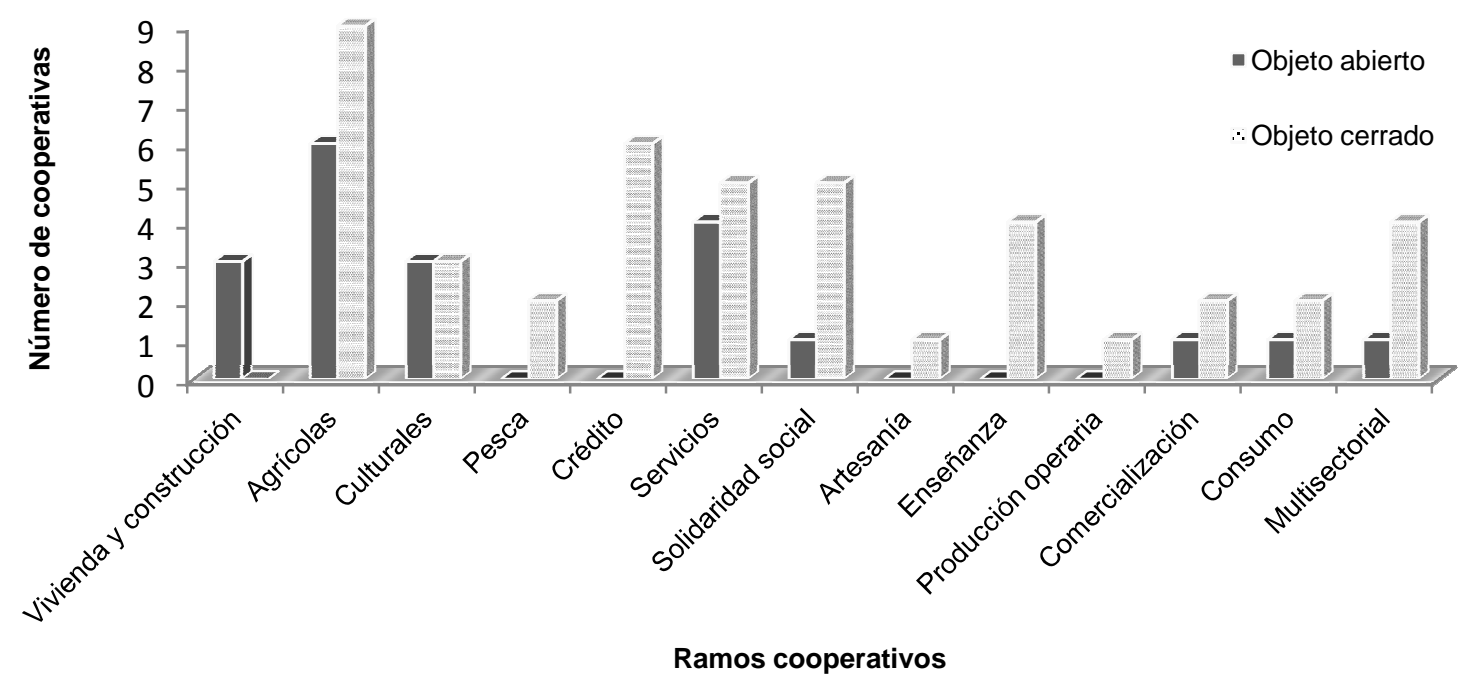

En la figura 1 se observa que la existencia de un "objeto abierto" es más común en ciertos ramos cooperativos que en otros. En dos ramos cooperativos, el crédito y la enseñanza, el fenómeno es casi inexistente. En el primer caso, la razón podría relacionarse con la legislación general de la actividad bancaria ${ }^{25}$, que impide a las entidades bancarias el ejercicio de otras actividades, mientras en el segundo, ello podría deberse a la especificidad de la

\footnotetext{
${ }^{25}$ Art. 4 del Decreto-Ley 298/92, de 31 de diciembre.
} 
actividad. Por otra parte, la ilustración 1 apunta tres ramos en los que la incidencia del fenómeno podría ser más acentuada: vivienda y construcción, agricultura, y servicios.

\subsubsection{Ejercicio de actividades no incluidas en el objeto}

Si en el punto anterior hemos tratado el problema de la definición del objeto estatutario de las cooperativas, en este punto se aborda la cuestión del ejercicio de actividades no previstas en los estatutos cooperativos, ya sea en simultáneo con el ejercicio del objeto estatutario o en exclusiva (se constató que el 5\% de las cooperativas inquiridas declaran no ejercer en absoluto la actividad que constituye su objeto principal). El ejercicio de actividades no comprendidas en el objeto estatutario debe ser interpretado como indicativo de una hibridación de la cooperativa, ya que tiene el propósito de aumentar la rentabilidad de la empresa y no de realizar el fin mutualista.

Sobre la primera situación, cabe mencionar una elevada incidencia del ejercicio de actividades no previstas en los estatutos ${ }^{26}$. De hecho, casi un $16 \%$ de las cooperativas declara desarrollar actividades no previstas en sus estatutos, ni como objeto principal ni como actividad complementaria, siendo que el $11 \%$ corresponde al "Grupo A" y el 5\% al "Grupo B". Cabe observar que estas actividades, según los datos resultantes de la encuesta realizada, alcanzan entre el $1 \%$ y el $58 \%$ del volumen de negocio de las cooperativas. Para las cooperativas del "Grupo A", este dato significa que esas cooperativas realizan actividades no exentas que pueden alcanzar un valor muy significativo con relación al volumen de negocio total. Ya para el "Grupo B", en cambio, las actividades no previstas en los estatutos realizadas generan rentas que, en principio, quedan exentas de impuesto si la cooperativa cumple con los requisitos para beneficiar de la exención. En cualquiera de los dos casos, los datos parecen indicar la emergencia de un fenómeno de hibridación de algunas cooperativas, que puede tener una expresión relevante y que debería ser tomado en consideración por la legislación tributaria.

\subsection{Las operaciones con terceros}

Para analizar la problemática de las "operaciones con terceros", hemos dividido el análisis según los dos regímenes o grupos fiscales cooperativos descritos con anterioridad ${ }^{27}$.

\footnotetext{
${ }^{26} \mathrm{Al}$ amparo del art. 9 do Código Cooperativo y del art. 142.1 al. d) del Código de Sociedades, el ejercicio de una actividad no comprendida en el objeto estatutario de la cooperativa constituye causa de disolución administrativa o por deliberación de la junta general.

${ }^{27}$ Ver punto III.2.
} 
Para el "Grupo A", el legislador utiliza el concepto de "operaciones con terceros" para delimitar la mutualidad, que goza de protección fiscal, de la "no mutualidad", que no goza de esa protección. ${ }^{28}$ Por esta razón, la cuantificación que resulte de los datos recogidos sobre la incidencia de "operaciones con terceros" en este grupo de cooperativas tiene un significado particular. Por otro lado, para el "Grupo B", el hecho de que lleven o no a cabo "operaciones con terceros" no tiene ninguna relevancia fiscal. Por ese motivo, el análisis de la incidencia de las "operaciones con terceros" en este grupo de cooperativas no adquiere un significado distinto del anterior, porque las cooperativas de este grupo no tienen, en principio, ninguna motivación fiscal para limitar el nivel de este tipo de operaciones, por lo que la comparación entre los datos arrojados por los dos grupos puede ser también reveladora.

Sobre este punto se preguntó a las cooperativas: si realizan o no "operaciones con terceros"; qué porcentaje alcanzan tales operaciones en el total de sus operaciones; si separan en su contabilidad las "operaciones con terceros" de las operaciones con socios; cómo hacen esa separación. Las primeras dos preguntas son las preguntas principales, mientras la tercera y la cuarta deberán ser analizadas como preguntas "filtro".

\footnotetext{
${ }^{28}$ La temática de la separación de las operaciones "con terceros" y "con socios" desde la perspectiva de sus efectos sobre los resultados disponibles fue tratada con interesantes resultados por LEJARRIAGA PÉREZ DE LAS VACAS, G., FERNÁNDEZ GUADÃNO, J. \& ITURRIOZ DEL CAMPO, J. (2005) Un estudio sobre la sensibilidad del coste asociado a la contabilización conjunta o separada de los resultados en la sociedad cooperativa, CIRIEC-España, Revista de Economía Pública, Social y Cooperativa, N. . 51, pp. 167-183. Sobre el mismo tema se puede ver también ITURRIOZ DEL CAMPO, J. (1999) Las operaciones con terceros en las sociedades cooperativas. La posibilidad de realizar una contabilización conjunta, REVESCO. Revista de Estudios Cooperativos, N. ${ }^{\circ} 67$, págs. 123-138.
} 
Figura 2. Operaciones con terceros en porcentaje del total y por ramos cooperativos del "Grupo A"

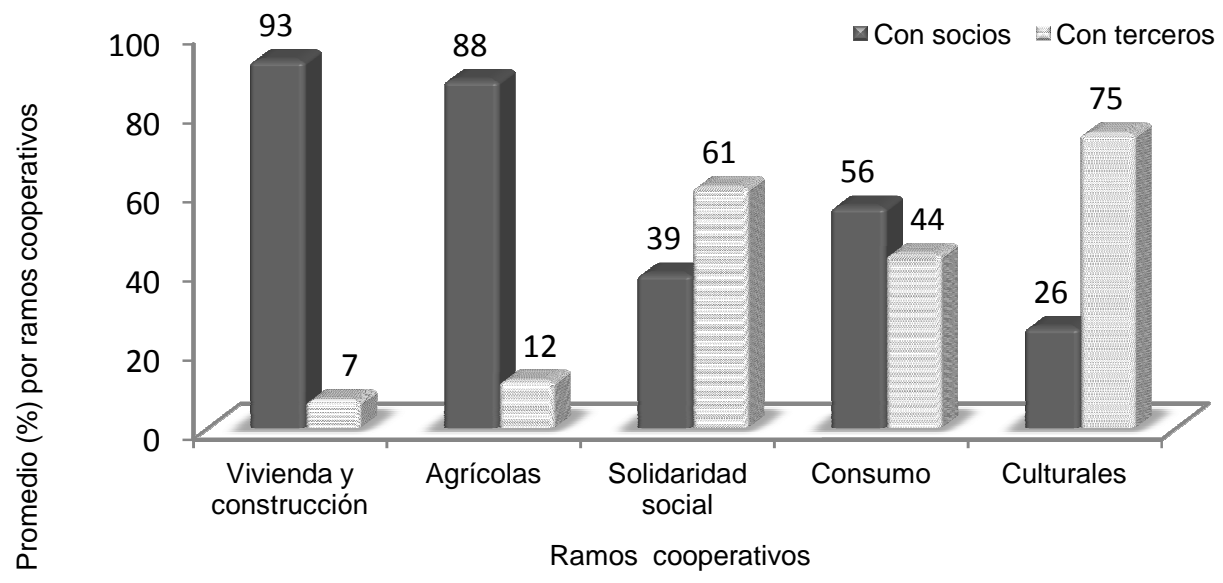

En lo que atañe a la primera cuestión, y limitándonos por ahora a las cooperativas del "Grupo A", se constató que el $97 \%$ de las cooperativas realizan operaciones con terceros. Además, para las cooperativas que declaran realizar operaciones con terceros, este tipo de operaciones representa un promedio del $30 \%$ del total de sus operaciones, es decir un nivel bastante elevado en el total del volumen de negocio, lo que ya sería indicativo, de por sí, de algún fenómeno de desmutualización de las cooperativas portuguesas, lo que supone una escasa eficacia de la normativa fiscal en incentivar la mutualidad, atendiendo a que estas operaciones quedan fuera del perímetro de la exención. Pero este dato, como se verá adelante, puede no reflejar la realidad del fenómeno en su verdadera dimensión.

La ilustración 2 sugiere que la incidencia de operaciones con terceros no se distribuye de manera uniforme en todos los ramos cooperativos del "Grupo A", y es muy superior en los ramos de solidaridad social (69\%), de cultura (75\%) y de consumo (44\%).

Ya para el total del "Grupo B", se constató que un 94\% de las cooperativas realizan operaciones con terceros. Los resultados de la encuesta enseñan que, en este grupo, las operaciones con terceros representan un $42 \%$ del total, contra el $58 \%$ que corresponde a las operaciones con socios, números que evidencian para este grupo un claro fenómeno de desmutualización. Por otro lado, cabe subrayar la significativa diferencia entre la incidencia de las "operaciones con terceros" en el "Grupo A" y en el "Grupo B", lo cual parece sugerir que el régimen de exención del "Grupo B" incentiva, o por lo menos propicia la desmutualización, más que el régimen del "Grupo A". 
Por otro lado, estos datos deben cotejarse con los resultados obtenidos en la pregunta filtro, “la contabilidad de la cooperativa está organizada de manera que separe las “operaciones con terceros" y las operaciones con socios (operaciones cooperativizadas)?". Curiosamente, un $36 \%$ de las cooperativas declararon que en su contabilidad no separan las "operaciones con terceros" de las operaciones con socios. De este porcentaje, un 9\% corresponde al "Grupo A", en que las "operaciones con terceros" quedan excluidas del ámbito de la exención. Sin duda estos números plantean varios problemas sobre el modo de interpretar los datos concernientes a la incidencia de las "operaciones con terceros". Uno de esos problemas, de incumbencia más directa para nuestro tema, es que, al no discriminar las operaciones con terceros en su contabilidad, las cooperativas del "Grupo A" imposibilitan que la administración tributaria les aplique correctamente el respectivo régimen fiscal. Esta dificultad para aplicar estrictamente la norma de exención resulta aún más agravada por el hecho de que las cooperativas no están obligadas, a diferencia de las sociedades mercantiles, a depositar los documentos contables en el registro mercantil ${ }^{29}$ (Meira, 2009: 100-102). Otro problema emergente de los datos recogidos, que no puede dejar de mencionarse, se refiere a la posible existencia de un fenómeno de desmutualización más extenso que el indicado por los datos.

\subsection{Estructura prevalentemente mutualista del factor trabajo}

La segunda línea divisoria principal utilizada en la normativa fiscal para separar la zona de la mutualidad, que debe ser protegida fiscalmente, de la zona de "no mutualidad", que no merecería dicha protección, está formada por la característica de la "estructura prevalentemente mutualista del factor trabajo", como ya se ha explicado ${ }^{30}$. Esta característica consiste en que un $75 \%$ de los trabajadores sean socios y que también un $75 \%$ de los socios sean trabajadores de la cooperativa. En este punto se buscó indagar, en primer lugar, en qué medida las cooperativas son capaces de cumplir este requisito o en qué medida cumplir dicho requisito resulta una finalidad relevante para las cooperativas del Grupo B. Como encuadramiento previo, hay que subrayar que se trata de un criterio diseñado originariamente para las cooperativas de producción operaria. Hoy día, la normativa fiscal ha extendido este mismo requisito a todos los ramos cooperativos que forman el llamado "Grupo B" 31 . Si bien se puede afirmar que el modelo fundamental de mutualidad para algunas de las cooperativas

\footnotetext{
${ }^{29}$ Artículos 3, al. n) y 4 del Código del Registro Comercial.

${ }^{30}$ Ver supra punto III.2.

${ }^{31}$ Ver supra punto III.2
}

REVESCO N 121 - Segundo Cuatrimestre 2016 - ISSN: 1885-8031 - www.ucm.es/info/revesco 
de este grupo continúa vinculado al factor trabajo, ello no se observa en todos los ramos por igual. Dentro de este grupo hay ramos cooperativos, como el crédito y la comercialización, en los que no consideramos que la mutualidad resida total o mayoritariamente en la "organización mutualista del factor trabajo".

Figura 3. No observancia del criterio de la estructura prevalentemente mutualista del factor trabajo por ramos cooperativos del Grupo B

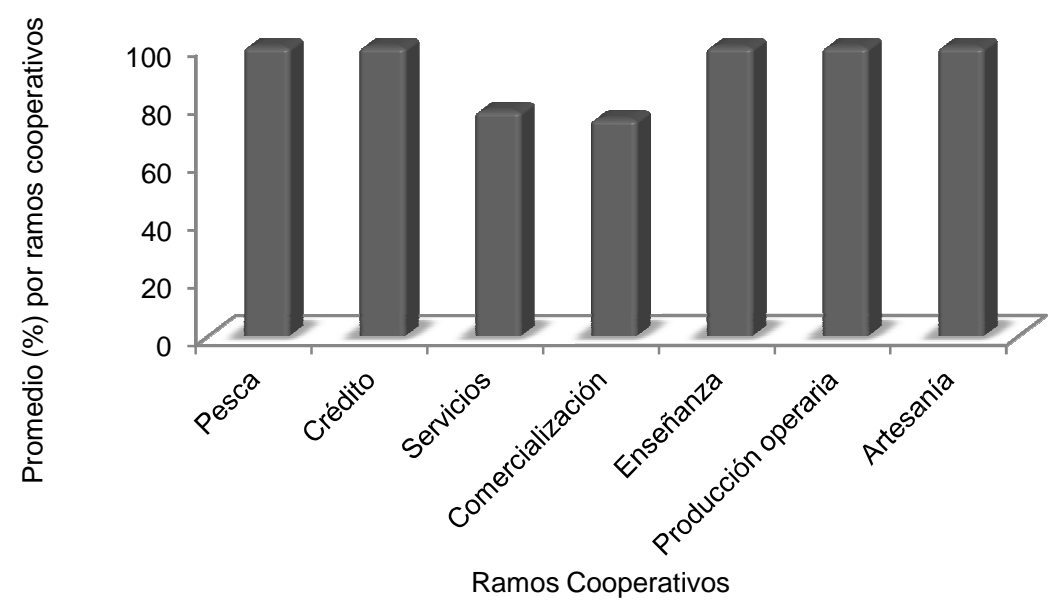

En lo que concierne a este punto, los resultados indican que sólo un reducido $4 \%$ de las cooperativas del grupo cumplen el criterio de la "estructura prevalentemente mutualista del factor trabajo", contra un $90 \%{ }^{32}$ que no lo cumplen. Como puede verse en la figura 3 , casi todos los sectores del grupo se acercan al 100\% de no observancia de tal criterio, si bien habrá que relativizar estos datos con relación a algunos sectores - la pesca, la producción operaria y la artesanía, en los cuales la muestra es particularmente reducida (estos ramos totalizaron 4 cuestionarios).

\section{CONCLUSIONES}

La primera conclusión que se extrae del presente estudio es que, por su dimensión, el fenómeno designado en este trabajo como "objeto abierto" (que consiste en no definir el objeto con la precisión suficiente que permita identificar las actividades específicamente ejercidas por la cooperativa, como sería necesario para una aplicación correcta del régimen de exenciones) no resulta conciliable con el régimen de exención establecido en la normativa tributaria. La elevada incidencia del fenómeno de "objeto abierto" impide en particular una aplicación correcta de los criterios relativos a las "operaciones con terceros" y a las

\footnotetext{
${ }^{32}$ La suma no llega al $100 \%$ debido al número de cooperativas que no contestaron la pregunta.
} 
"operaciones ajenas al fin propio de la cooperativa". Por otra parte, la existencia de un "objeto abierto" hace que no sea posible determinar con rigor en qué régimen de exención debe encuadrarse la cooperativa. ${ }^{33}$

Con respecto al criterio de la "estructura prevalentemente mutualista del factor trabajo", también utilizado en la normativa fiscal para trazar la frontera entre mutualidad y "no mutualidad", los resultados muestran que una fracción muy reducida de las cooperativas lo cumplen, lo que significa que la aplicación estricta de la normativa fiscal excluiría a la mayoría de las cooperativas del "Grupo B” del régimen de exención.

Dentro de las hipótesis que establecimos como base de trabajo, habría que concluir que se comprueban tanto la segunda como la tercera hipótesis, en grados distintos y con incidencia diferente en los dos grupos de ramos cooperativos señalados.

Las cooperativas del "Grupo B" (de comercialización, de crédito, de producción operaria, de artesanía, de pesca, de enseñanza y de servicios) muestran una tendencia acentuada para apartarse del padrón tributario actual de mutualidad, al no observar el criterio de la "estructura prevalentemente mutualista del factor trabajo", lo cual supone un elevado grado de ineficacia de la norma de exención para proteger el modelo empresarial mutualista. A pesar de que tal criterio parece estar particularmente desajustado con relación a algunos sectores, como el crédito, los datos recogidos muestran una elevada tasa de inobservancia en todos los ramos.

También con relación al "Grupo B", los números sobre "operaciones con terceros" confirman claramente la emergencia del fenómeno de desmutualización. Los resultados apuntan por tanto para un evidente desajuste entre la norma fiscal de exención aplicable a este grupo y el fin perseguido por la misma.

En cuanto al "Grupo A", los datos recogidos indican, prima facie, un nivel más elevado de coincidencia con los criterios que delimitan la zona de protección físcal operaciones con socios y actividades conectadas con el fin propio de la cooperativa. Sin embargo, el hecho de que un porcentaje elevado de cooperativas de este grupo declare no hacer una separación contable de las operaciones con terceros impone reservas sobre la validez de una conclusión acerca del grado de sintonía entre los criterios fiscales y la realidad cooperativa. Parece confirmarse así, sobre todo con respecto a este grupo, la tercera hipótesis

\footnotetext{
${ }^{33}$ Art. 66.1 del Estatuto de los Beneficios Fiscales.
} 
planteada, de un desajuste de carácter técnico entre el régimen fiscal y la realidad cooperativa. Si bien es cierto que la legislación portuguesa no impone una separación contable entre las operaciones con miembros y con terceros, creemos poder concluir que hace falta un control externo más eficaz de los aspectos contables de las cooperativas, a fin de que el régimen de protección fiscal pueda cumplir su función.

\section{BIBLIOGRAFÍA}

ABREU, J. M. (2012) Curso de Direito Comercial, Vol. I, 8a ed., Coimbra: Almedina. 423 p. ISBN: 978-972-40-4677-8.

AGUIAR, N. (2014) A tributação do rendimento das cooperativas em Portugal, Revista de Cooperativismo e Economia Social, № 36, 2013/2014, pp. 55-80.

ALGUACIL MARÍ, M. P. (2010) Condicionantes del régimen de ayudas de Estado en la fiscalidad de cooperativas, CIRIEC-España, Revista de Economía Pública, Social y Cooperativa, $\mathrm{N}^{\circ} 69$, pp. 27-52.

ARANA LANDÍN, S. (2012) Régimen fiscal de las cooperativas. Bilbao: Universidad del País Vasco. 230 p. ISBN: 978-84-9860-720-8

BOETTCHER, E. (1981) Las cooperativas en una economía de mercado. Buenos Aires: Intercoop. 179 p. s/ISBN. Traducción de R.VILLEGAS VELÁSQUEZ.

CALVO ORTEGA, R. (2005) La sociedad cooperativa europea. Un paso adelante en la fiscalidad de la economía social. En: CALVO ORTEGA, R. Fiscalidad de las entidades de economía social: cooperativas, mutuas, sociedades laborales, fundaciones, asociaciones de utilidad pública, centros especiales de empleo, empresas de inserción social. Madrid: Civitas, pp. 65-73. ISBN: 84-470-2354-0.

CHAMBERS, E. (1947) Should the earnings of co-operative associations be made subject to the federal income tax? British Columbia: University of British Columbia. 148 p. s/ ISBN.

CUSA, E. (2013) Le forme di impresa private diverse dalle società lucrative tra aiuti di Stato e Costituzionieconomicheeuropee, Costituzioni economiche europee. Torino: G.

Giappichelli Editore. 139 p. ISBN: 978-88-348-7833-0

DILI, A. (2007) L'Iresnellesocietàcooperative. Mutualità, ristorni, agevolazioni. Milán: Giuffrè Editore. 284 p. ISBN: 8814128294.

DUARTE P. (2012): Reflexos jurídico-obrigacionais da cooperatividade nos negocios jurídicos celebrados pelas cooperativas de habitação e construção. En: MEIRA, D. 
Jurisprudência Cooperativa Comentada. Lisboa: Imprensa Nacional Casa da Moeda, pp. 479-497. ISBN: 978-972-27-2118-9.

ELENA DÍAS, F. (1990) Comentarios al proyecto de Ley sobre Fiscalidad Cooperativa. REVESCO, Revista de Estudios Cooperativos, $\mathrm{N}^{\circ}$ 58, pp. 79-90.

FAJARDO GARCÍA, I. (1997) La gestión económica de la cooperativa: responsabilidad de los socios. Madrid: Tecnos. 246 p. ISBN: 84-309-3015-9.

FAJARDO GARCÍA, I. G. (2013) La especificidad de las sociedades cooperativas frente a las sociedades mercantiles y la legitimidad de su particular régimen jurídico y fiscal según el tribunal de justicia de la Unión Europea, Revista de Derecho Mercantil, № 288, pp. 189222.

HINOJOSA TORRALVO, J. J. (2010) Fiscalidad y financiación de las cooperativas:¿a qué juega la Unión Europea? CIRIEC-España Revista de Economía Pública, Social y Cooperativa, $\mathrm{N}^{\mathrm{o}} 69$, pp. 73-89.

ISH, D. (1975) Some Aspects of the Taxation of Canadian Co-operatives, Canadian Tax Papers, No 57, pp.42-78.

ITURRIOZ DEL CAMPO, J. (1999) Las operaciones con terceros en las sociedades cooperativas. La posibilidad de realizar una contabilización conjunta, REVESCO, Revista de Estudios Cooperativos, N. ${ }^{\circ}$ 67, pp. 123-138.

LEJARRIAGA PÉREZ DE LAS VACAS, G., FERNÁNDEZ GUADÃNO, J. \& ITURRIOZ DEL CAMPO, J. (2005) Un estudio sobre la sensibilidad del coste asociado a la contabilización conjunta o separada de los resultados en la sociedad cooperativa, CIRIECEspaña, Revista de Economía Pública, Social y Cooperativa, N. ${ }^{\circ}$ 51, pp. 167-183.

LLOBREGAT HURTADO, M. L. (1990) Mutualidad y empresas cooperativas. Barcelona: Bosch. 464 p. ISBN: 84-7698-143-0.

MARTÍNEZ SEGOVIA, F.J. (2005) La relación cooperativizada entre la sociedad cooperativa y sus socios: naturaleza y régimen jurídicos, Revista de Derecho de Sociedades, $\mathrm{N}^{\mathrm{o}} 25$, pp. 203-234.

MEIRA, D. (2009) O regime económico das cooperativas no direito português: O capital social. Porto: Vida Económica. 390 p. ISBN: 978-972-788-292-2.

MEIRA, D. (2011) O quadro jurídico-constitucional do cooperativismo em Portugal, Cooperativismo e Economia Social, № 33, pp. 31-46.

MEIRA, D. (2012a) Revisitando o problema da distinção entre excedente cooperativo e lucro societário, En: DUARTE, R. P., VASCONCELOS, P. P. y ABREU, II Congresso de 
Direito das Sociedades em Revista. Coimbra: Almedina, pp. 353-376. ISBN: 978-972-404962-5.

MEIRA, D. (2012b) As operações com terceiros no direito cooperativo português. En: MEIRA, D.Jurisprudência Cooperativa Comentada. Obra coletiva de comentários a acórdãos da jurisprudência portuguesa, brasileira e espanhola. Lisboa: Imprensa Nacional - Casa da Moeda, pp. 413-425. ISBN: 978-972-27-2118-9.

MEIRA, D. (2013a) A relevância do cooperador na governação das cooperativas, Cooperativismo e Economia Social, № 35, p. 9-35.

MEIRA, D. (2013b) A Lei de bases da economia social portuguesa: do projeto ao texto final», CIRIEC-España, Revista Jurídica de Economía Social y Cooperativa, № 24, p. 21-52, ISSN 1577-4430.

NABAIS, J. C. (1998) O dever fundamental de pagar impostos. Coimbra: Almedina. 746 p. ISBN: 9724011151.

NAMORADO, R. (2000) Introdução ao Direito Cooperativo. Para uma expressão jurídica da cooperatividade. Coimbra: Almedina. 349 p. s/ISBN.

PANIAGUA ZURERA, M. (2005) Las sociedades cooperativas. Las sociedades mutuas de seguros y las mutualidades de previsión social. En: JIMÉNEZ SÁNCHEZ, G. Tratado de Derecho Mercantil, T. XII, Vol. 1, Barcelona: Marcial Pons, pp. 659-686, ISBN: 84-9768214-9.

PAOLUCCI, L. F. (2004) Le societàcooperative dopo la riforma. Appendice. Commentario breve agli articoli da 2511 a 2545 octiesdecies del c.c. e al d.lgs. 2 agosto 2002, n. 220, in tema di vigilanza sulle cooperative. Padua: Cedam. 376 p. ISBN: 978-88-13-25163-5

PEREIRA, M. H. et al. (1998) Reavaliação dos benefícios fiscais. Relatório do grupo de trabalho constituído pelo Despacho núm. 130/97-XIII do Ministro das Finanças, Cadernos de Ciência e Técnica Fiscal, № 180, pp. 5-341.

PETROU, T. (2013) Canada. En: CRACOGNA, D., FICI, A. y HENRY, H. International Handbook of Cooperative Law. Heidelberg / Nueva York / Dordrecht / Londres: Springer, pp. 289-316, ISBN: 978-3-642-30128-5.

PIGOU, A. C. (1920) Co-operative Societies and Income Tax, The Economic Journal, Vol. $30, \mathrm{~N}^{\mathrm{o}} 118$, pp.156-162.

RODRIGO RUIZ, M. A. (2003) Mandato constitucional de fomento y fiscalidad de lascooperativas, CIRIEC-España, Revista de economía pública, social y cooperativa, $\mathrm{N}^{\circ}$. 47, pp. 199-219. 
RODRIGO RUÍZ, M. A. (2010) Consideraciones sobre el régimen fiscal de las cooperativas. Problemas actuales y líneas de reforma, CIRIEC-España, Revista de Economía Pública, Social y Cooperativa, № 69, pp. 9-25.

ROMERO CIVERA, A. (2010) La fiscalidad aplicada a cooperativas en Europa y la reforma del régimen fiscal en España, CIRIEC-España, Revista de Economía Pública, Social y Cooperativa, № 69 , pp. 91-118.

SARINA, T. (2013) Australia. En: CRACOGNA, D., FICI, A. y HENRY, H. International Handbook of Cooperative Law, Heidelberg / Nueva York / Dordrecht / Londres: Springer, pp. 207-229, ISBN: 978-3-642-30128-5.

SNAITH, I. (2013) United Kingdom. En: CRACOGNA, D., FICI, A. y HENRY, H. International Handbook of Cooperative Law, Heidelberg / Nueva York / Dordrecht / Londres: Springer, pp. 735-757, ISBN: 978-3-642-30128-5.

STRACKE, B. (1997) Besteuerung von Genossenschaften in der Europäischen Union. Bilefeld: Erich Schmidt. 252 p. ISBN: 978-3503041169.

TEJERIZO LÓPEZ, J. M. (2010) Algunas reflexiones sobre el régimen fiscal de las cooperativas. CIRIEC-España, Revista de Economía Pública, Social y Cooperativa, ${ }^{\circ}$ 69, pp. 53-72.

TIPKE, K. \& LANG, J. (2005) Steuerrecht, $18^{a}$ ed. Colonia: Otto Schmidt. 1118 p. ISBN:3504-20138-X

VARGAS VASSEROT, C. (2006) La actividad cooperativizada y las relaciones de la cooperativa con sus sócios y con terceros, Madrid: Aranzadi. 200 p. ISBN: 84-8355-0075.

VARGAS VASSEROT, C. y AGUILAR RUBIO, M. (2004) Las operaciones de la cooperativa con terceros y la infundada limitación de las mismas por su tratamiento fiscal privilegiado. REVESCO. Revista de Estudios Cooperativos, № 83, pp. 115-140.

VERRUCOLI, P. (1962) Cooperative (Imprese). En: Enciclopedia del Diritto, Milán: GiuffrèEditore, pp. 549-609, ISBN: 8814147639. 